\title{
On the Impact of Fundamentals, Liquidity and Coordination on Market Stability*
}

\author{
Jón Daníelsson \\ Francisco Peñaranda \\ London School of Economics Universitat Pompeu Fabra
}

January 2007

\begin{abstract}
Complex interactions between fundamentals and liquidity during unstable periods in financial markets are succinctly modeled with coordination games. We propose a flexible framework to estimate such a model and use the efficient method of moments as estimation procedure. We illustrate the model by using exchange rates from the yendollar carry trade induced uncertainty in 1998, interest rate spreads and global market volatility. The model fits the data well, with evidence of low information disparities, the market is generally very deep, where global volatility is more important than fundamental uncertainty in the determination of liquidity. There is clear evidence of asymmetry between the buy and sell sides of the market.
\end{abstract}

\footnotetext{
${ }^{*}$ Corresponding author Jón Daníelsson. We would like to thank Amil Dasgupta, Irma Clots-Figueras, Antonio Díez, Masazumi Hattori, Ron Gallant, Frank Heinemann, Ángel León, Guillaume Plantin, Enrique Sentana and Hyun Shin for very useful comments and suggestions. We acknowledge the financial support of the EPSRC grant no. GR/S83975/01. Our papers can be downloaded from www.RiskResearch.org.
} 


\section{Introduction}

Recent episodes of financial market instability have highlighted the complex interaction between economic fundamentals and liquidity, where the reaction of agents to information arrival and their expectation of the behavior of other agents has the potential to endogenously decrease market stability. Since the resulting trading decisions of agents reinforce each other they are naturally modeled by coordination game theoretic frameworks, such as the global games models of Morris and Shin (1998) and subsequent papers. Unfortunately, such models do not lend themselves naturally to estimation. By contrast, extant empirical models of financial instability are generally purely statistical, which limits their economic interpretations. A quantitative study of market stability requires the direct estimation of the deep parameters of an underlying theoretical model, which provides our basic motivation. Below, we develop and estimate a coordination game model of market instability only requiring publicly available data. Ultimately, this estimation both provides empirical tests of the underlying theoretical model as well as guidance for policymaking and risk management.

Most attempts of applying coordination games to financial data have focused on currency crises, and assume common knowledge of economic fundamentals, such as the $2^{\text {nd }}$ generation currency crisis models, (see e.g. Obstfeld, 1986). Common knowledge generally implies multiple equilibria and sunspots, such as the Markov-switching model of Jeanne and Masson (2000). Unfortunately, in the absence of ad-hoc assumptions, multiple equilibria frustrates empirical implementations. However, by making the plausible assumption of asymmetric information a unique equilibrium can be found, facilitating empirical investigation. Such models, (see e.g. Morris and Shin, 1998; Goldstein and Pauzner, 2005; Morris and Shin, 2004), termed global games models, provide the theoretic background to our model. Several authors have estimated the role of information for the propensity for speculative attacks using reduced form specifications motivated by global games, and find indirect empirical support for the theory. ${ }^{1}$

In contrast, our objective is the estimation of deep parameters, imposing structure on the econometric model, providing direct testability of theory, and interpretation of results. This enables us to measure the interaction between fundamentals and liquidity and its implications for market (in)stability. A key challenge is the joint modelling of liquid and illiquid times in a time series setting, while keeping the model structure sufficiently simple and par-

\footnotetext{
${ }^{1}$ See inter alia Prati and Sbracia (2002), Tillmann (2002) and Metz and Michaelis (2003).
} 
simonious without loosing theoretical insight.

We develop our model in two stages, first focusing on the endogenous reaction of traders to a particular environment of fundamentals and liquidity. We consider a market for financial assets focusing on the behavior of agents who trade for speculative reasons, termed strategic agents. The strategic agents, representing e.g. proprietary trading desks and hedge funds, face the residual demand of the rest of market participants, or nonstrategic agents. While each strategic agent exerts a negligible price impact, as a group they can have a significant price impact when that residual demand is not infinitely elastic, i.e. at times of liquidity tensions. Each strategic agent takes this fact into account when taking trading decisions and those decisions are mutually reinforcing.

This results in a one period global game with a unique equilibrium, which is brought to data as a sequence of one shot games given the short-termism of strategic agents. This is similar in spirit to Foster and Viswanathan (1995) who apply the one trading round version of Kyle (1985) to a time series. Since it is unrealistic to assume that the distribution of fundamentals and liquidity tensions is constant over time, we also model the dynamic evolution of relevant state variables linked to fundamentals and market liquidity.

Our notion of liquidity relates to Grossman and Miller (1988), which is based on inventory risk. ${ }^{2}$ Price changes are determined by fundamentals and uncertainty, the nonstrategic agents' liquidity needs and risk aversion, and the edogenous reaction of strategic agents to such environment. By means of nonstrategic agents' liquidity shocks (an inelastic component in the residual demand they provide), we explicitly allow for, but do not require, asymmetry on the buy and sell sides, which in turn is a testable hypothesis. Given our ultimate objective of estimation, we model illliquidity with publicly available data.

Unfortunately, it is not possible to estimate our model with standard techniques such as maximum likelihood or GMM due to the highly non-linear nature of the model and the presence of dynamic latent variables. However, since the model can be easily simulated, we employ the efficient method of moments proposed by Gallant and Tauchen (1996, 2002). This procedure identifies the optimal data moments and it is an alternative to arbitrarily identifying a set of moments and then proceeding with GMM or simulated method of moments. The use of optimal moments delivers efficiency close to

\footnotetext{
${ }^{2}$ Another coordination game that follows this notion of liquidty is Morris and Shin (2004). However, they focus on loss limits as a source of coordination and asymmetric information, which is not the case in our model.
} 
or the same as maximum likelihood estimation. ${ }^{3}$

Our empirical application is the yen dollar market in 1998, where following a long period of successful carry trades, in a span of couple of days a substantial number of those were reversed, triggering previously unforeseen levels of appreciations of the yen against the dollar. This was to a considerable extent triggered by events unrelated to the Japanese market, e.g. the aftermath of the Russian and LTCM crisis, and yield curve adjustments in the run-up to the adoption of the Euro. The slow buildup and rapid reversal of carry trades implies a particular pattern for prices, sometimes denoted as up by the escalator, down by the elevator, and constitutes a testable hypothesis.

We estimate the model with daily yen dollar exchange rate returns from 1992 to 2004, enabling us to include both time periods with and without turmoil. Furthermore, we estimate the model in three subsamples, enabling us to focus on the different market conditions throughout the sample period. In addition to estimating the model parameters, we illustrate the results by means of simulations. We employ global volatility, and the interest rate spread between Japan and US as proxies for payoff uncertainty and fundamentals.

Our model fits the data well, it is not statistically rejected and all parameters are significant. There is evidence of low information disparities amongst agents, the market is generally very deep, where global volatility is more important than fundamental uncertainty in the determination of liquidity. We find clear evidence of asymmetry between the buy and sell sides of the market, with clear escalator-elevator effects in prices where low levels of market illiquidity have a bigger effect on the buy side, while higher levels of illiquidity are primarily felt on the sell side. These effects are especially pronounced in the second subsample which contains the main crisis event.

\section{Returns under Liquidity Tensions}

Consider a market for a financial security, with two categories of agents, strategic agents and nonstrategic agents. The strategic agents trade for short term speculative reasons, represent institutions such as proprietary traders and hedge funds, and play a coordination game. The remainder of market participants, or nonstrategic agents, are represented by a residual demand

\footnotetext{
${ }^{3}$ The EMM method has seen a wide range of applications, mostly in the area of asset pricing. There are applications to stochastic volatility such as Chernov and Ghysels (2000) and Chernov et al. (2003); applications to interest rates such as Dai and Singleton (2000), Bansal and Zhou (2002), and Ahn et al. (2002); and applications to exchange rates such as Gallant and Long (1997) and Chung and Tauchen (2001)
} 
that defines the environment of fundamentals and liquidity that strategic traders face.

We present our model in two stages, below we focus on the endogenous reaction of strategic traders to a particular environement by means of a one-period theoretic model. We subsequently develop an econometric model for the dynamic evolution of that environment, which is required for the estimation of the coordination game model with time series data.

\subsection{Asset Return Decomposition}

There is a continuum of strategic agents uniformly distributed on the interval $[0,1]$. Each strategic agent makes a binary decision, of either buying or selling one unit of the asset. Indicate the fraction of agents selling by $\lambda \in[0,1]$.

We represent nonstrategic agents by residual demand that is linear in the asset price. We impose in Appendix A a market clearing condition by the end of trading period that equalizes the residual demand of nonstrategic agents to the net supply of strategic agents, where we can express the observed rate of return on the risky asset as:

$$
r=v-\lambda c^{-}+(1-\lambda) c^{+}
$$

that is, $r$ is modeled as the sum of two components,

$$
\text { Observed return }=\text { fundamental return }+ \text { strategic return, }
$$

where the strategic return is the net price impact of the strategic agents, aggregating the buy price impact $(1-\lambda) c^{+}$, and the sell price impact $\lambda c^{-}$, with $c^{+}, c^{-} \geq 0$.

Liquid times are given by $c^{+}=c^{-}=0$ implying $r=v$. Hence the fundamental return $v$ is the one period rate that would be observed if the residual demand was perfectly elastic. It is driven by asset fundamentals, the expected liquidation value of the asset under study, and hence represents the exogenous arrival of information during the period. It is assumed to be normally distributed

$$
v \sim N\left(m, \alpha^{-1}\right),
$$

where $\alpha$ is the precision of the fundamental return, and $m$ is its mean. $m$ is the expectation of asset fundamentals at the beginning of the period, while asset fundamentals refer to the expectation at the end of the period of an asset's liquidation value. 
Liquidity tensions are represented by at least one of $\left(c^{+}, c^{-}\right)$higher than 0 . Therefore, we can interpret $\left(c^{+}, c^{-}\right)$as a measure of (lack of) liquidity or market depth on the buy and sell side. By contrast, in most standard one period models, either $c^{+}$or $c^{-}$is zero, since such models soley focus on the effect of agents one direction impact, e.g. a decision to launch a speculative attack or not. Since our ultimate objective is to estimate the model from time series data, we model both buying and selling decisions.

While the price impact of an individual strategic agent is negligible, they exert significant price impact if a sufficient number of strategic agents make the same trading decision during times of illiquidity. This in turn gives rise to strategic behavior where the equilibrium value of $\lambda$ is the solution of a game, denoted by $\lambda^{e}$.

\subsection{Trading Decisions of Strategic Agents}

Each strategic agent is a short-term speculator that trades depending on her expectation of the asset return. She makes a binary trading decision to sell or buy one the unit of the asset depending on her expectation of the fundamental return, as well as the trading decisions of other strategic agents, neither of which they observe prior to trading.

Each strategic agent $i$ buys one unit of the asset if she expects a price increase by the end of the period, and sells otherwise, i.e. if

$$
\mathrm{E}\left(r \mid \Omega_{i}\right)>0
$$

and sells one asset unit otherwise, where $\Omega_{i}$ means information available at the beginning of period. If agent $i$ expects a high $\lambda$ she will go short, while if she expects a low $\lambda$ she will go long.

The strategic agents only have access to incomplete information. They know both the price impacts, $\left(c^{+}, c^{-}\right)$and the parameters of the fundamental returns distribution $(m, \alpha)$, but they do not observe $v$, which depends on the arrival of information during the period, before their trading decision. However, each strategic agent $i$ receives a private signal $x_{i}$ :

$$
x_{i}=v+\varepsilon_{i}, \quad \varepsilon_{i} \stackrel{\mathrm{iid}}{\sim} N\left(0, \beta^{-1}\right)
$$

where the signal noise is independent and identically distributed across agents. The parameter $\beta$ is the signals' precision and is known by all agents. It is a measure of information disparities among the strategic agents. 
Hence the relevant information set of each agent $i$ for making a trading decision is:

$$
\Omega_{i}=\left(m_{i}, \alpha, \beta, c^{+}, c^{-}\right),
$$

where $m_{i}$ is the posterior mean of $v$ for this agent after observing $x_{i}$, a linear combination of $\left(m, x_{i}\right)$.

\subsection{Market Equilibrium}

We focus on monotone strategies whereby a strategic agent buys if her private signal exceeds $x_{o}$, and sells otherwise, implying that $x_{o}$ is the signal of the marginal agent, who has information set $\Omega_{o}$, and is indifferent between buying and selling. By applying a law of large numbers to the cross section of agents, the equilibrium fraction of agents selling, denoted by $\lambda^{e}$, is

$$
\lambda^{e}=\operatorname{Pr}\left(x<x_{o} \mid \Omega_{o}, v\right)
$$

where $x$ represents the distribution of signals across agents. The following Lemma characterizes $\lambda^{e}$.

Lemma 1 There is a unique equilibrium in the coordination game if and only if

$$
\frac{\alpha^{2}(\alpha+\beta)}{\beta(\alpha+2 \beta)} \leq \frac{2 \pi}{\left(c^{-}+c^{+}\right)^{2}},
$$

such that

$$
\lambda^{e}=\Phi\left[\sqrt{\beta}\left(x_{o}-v\right)\right],
$$

where $x_{o}$ is the signal of the agent that is indifferent between buying and selling.

This follows directly ${ }^{4}$ from showing that the condition in Lemma 1 is a sufficient and necessary condition for a unique symmetric equilibrium where every trader buys the asset if and only if her signal is such that $x<x_{o}$, where $x_{o}$ is given by $x_{o}=\beta^{-1}\left[(\alpha+\beta) m_{o}-\alpha m\right]$ with $m_{o}$ solving the fixed point equation

$$
m_{o}+c^{+}=\left(c^{-}+c^{+}\right) \Phi\left[\sqrt{\omega} \frac{\alpha}{\beta}\left(m_{o}-m\right)\right] .
$$

\footnotetext{
${ }^{4}$ The corresponding proof is available upon request from the authors.
} 
Finally, this switching strategy is the only one that survives iterated dominance. That is, there cannot be any other equilibrium when the symmetric one exists.

We can interpret the uniqueness condition as a lower bound on $\beta$ for given $\alpha$ and price impacts. That is, a unique equilibrium requires private information to be precise enough with respect to public information. ${ }^{5}$ Note that $\lambda^{e}$ is monotonically decreasing in $v$, providing a clear connection between fundamentals and equilibrium, and it is also a function of liquidity tensions given by $\left(c^{+}, c^{-}\right)$.

Note that, even though we wrote observed returns as the sum of fundamental and strategic returns in (2), $\lambda^{e}$ defines a complex interaction of $\left(m, \alpha, \beta, c^{+}, c^{-}\right)$in the defintion of $r$. That is, we find a potentially complex interaction of fundamentals and liquidity. To clarify such interaction, consider the special case where $\beta \rightarrow \infty$, i.e. the signal precision is unbounded.

In this case, every agent will do the same, buying or selling, depending on the realization of fundamental returns and price impacts. The marginal agent's beliefs about $\lambda^{e}$ in the limit are uniform in $[0,1]$ whatever the signals' distribution, which can be interpreted as the highest strategic uncertainty when $\beta \rightarrow \infty$ even though fundamental uncertainty becomes negligible. Hence, the marginal agent expects half of the agents to sell and her signal is $x_{o}=0.5\left(c^{-}-c^{+}\right)$. We arrive to the following corollary:

Corollary 2 If $\beta \rightarrow \infty$ there is a unique equilibrium in the coordination game with

$$
\lambda^{e}=\mathrm{I}\left[v<0.5\left(c^{-}-c^{+}\right)\right]
$$

where $\mathrm{I}[\cdot]$ denotes the indicator function.

Now $\lambda^{e}$ is not a continuous function of $v$ due to small information disparities, i.e., market outcomes are more extreme in this context. In addition, if liquidity tensions on the sell side are much higher than on the buy side, $c^{-}>>c^{+}$, then even a relatively high $v$ might not be enough to avoid $\lambda^{e}=1$. And vice versa, if liquidity tensions on the buy side are much higher than on the sell side $c^{+}>>c^{-}$then even a relatively low $v$ might not be enough to avoid $\lambda^{e}=0$. On the other hand, if $c^{-}=c^{+}$then $\lambda^{e}=\mathrm{I}[v<0]$, which is independent of liquidity tensions. However, this irrelevance of symmetric liquidity for trading decisions is not the case for bounded $\beta$.

\footnotetext{
${ }^{5}$ The asymmetric information among the strategic agents helps to pin down a unique equilibrium. If $v$ was common knowledge then there would be multiple equilibria for some values of $v$.
} 
Lemma 1 implies that the observed return in equilibrium is

$$
r=\left(v+c^{+}\right)-\left(c^{-}+c^{+}\right) \Phi\left[\sqrt{\beta}\left(x_{o}-v\right)\right],
$$

while the corollary shows that, under unbounded precision, it behaves like

$$
r=\left(v+c^{+}\right)-\left(c^{-}+c^{+}\right) \mathrm{I}\left[v<0.5\left(c^{-}-c^{+}\right)\right] .
$$

\section{Econometric Model of Fundamentals and Liquidity}

Above we focused on one-period trading decisions, however, in order to estimate the model with available time series data it is necessary to extend it to a time series setting. For ease of exposition, we think of each period as one day, but of course the model applies to other data frequencies. It is now necessary to index the model variables by time, so we write (1) as:

$$
r_{t}=v_{t}-\lambda_{t} c_{t}^{-}+\left(1-\lambda_{t}\right) c_{t}^{+}
$$

We assume the nonstrategic traders behave in a such a way that (9) holds and the distribution of $v_{t}$ is Gaussian conditional on the fundamental, in the dynamic framework the expected future dividends discounted at the risk-free rate, and uncertainty $\left(m_{t}, \alpha_{t}^{-1}\right)$

$$
v_{t} \sim N\left(m_{t}, \alpha_{t}^{-1}\right)
$$

which reflects the exogenous information arrival, that is, the strategic agents' uncertainty about the end of period fundamentals.

The equilibrium value of $\lambda_{t}$ in Lemma 1 remains the same in the time series model because strategic agents are short-term speculators who do not take into account future returns in current trading decisions. Short-term goals are a clear feature of many participants in financial markets due to reasons such as loss limits.

In sum, the time series model is a sequence of one-shot games of the type discussed above. Nevertheless, it is unrealistic to expect the environment of fundamentals and liquidity defined by the state variables $\left(m, \alpha, \beta, c^{+}, c^{-}\right)$ to remain constant over time, and consequently we need to model them as stochastic variables. 


\subsection{Fundamental Returns}

The fundamental mean is linked to a predictor of fundamentals that is observable by strategic agents before they take trading decisions. Specifically, a lagged proxy of fundamentals $z_{t-1}^{\mathrm{F}}$. In our case, we employ a linear transformation

$$
m_{t}=\gamma_{0} z_{t-1}^{\mathrm{F}}
$$

for a real number $\gamma_{0}$. The actual proxy for fundamentals used in the econometric application depends on the particular market under study and the frequency of observations. If no fundamental proxies are available we can model them as a latent stochastic process, or even a fixed parameter. We will illustrate these alternatives in our empirical application below.

In addition, we need to model the volatility of fundamental returns, and in this we are guided by both stylized facts of financial returns and theoretical considerations. In particular, we assume that the noise component of $v_{t}$ follows a stochastic volatility (SV) model, proposed originally by Clark (1973), with Taylor (1986) developing the dynamic version of the SV model that underpins subsequent developments. The SV model has a natural continuous time representation and as such has application in option pricing, where the exogenous volatility process can be interpreted as news arrival. Recently asset prices implications with stochastic volatility in fundamentals have been considered, e.g. by Tauchen (2005).

Following a standard SV model, the fundamental return precision $\alpha_{t}^{-1}$ is a stationary $\mathrm{AR}(1)$ process in logs with normal innovations:

$$
-\ln \alpha_{t}-\gamma_{1}=\gamma_{2}\left(-\ln \alpha_{t-1}-\gamma_{1}\right)+\gamma_{3} u_{t}, \quad u_{t} \stackrel{\mathrm{iid}}{\sim} N(0,1), \quad\left|\gamma_{2}\right|<1,
$$

where $u_{t}$ and the Gaussian innovation of $v_{t}$ are independent. This is consistent with the assumed information structure in trading decisions, where knowledge of $\alpha_{t}$ by strategic agents does not give them extra information about the level of $v_{t}$.

\subsection{Private Signals}

Another relevant dimension of fundamental returns is the private information that strategic traders have about them. A consequence of (7) in Lemma 1 is that because $\left(\alpha_{t}, \beta_{t}\right)$ and $\left(c_{t}^{+}, c_{t}^{-}\right)$change over time, the uniqueness condition needs to be satisfied at each point in time in the econometric model. Therefore, the stochastic process for $\beta_{t}$ should satisfy that constraint. Denote the 
lower bound by $b_{t}$, then a stochastic $\beta_{t}$ that satisfies (7) is:

$$
\ln \left(\beta_{t}-b_{t}\right)=\eta,
$$

where $\eta$ is a real number that defines $\beta_{t}$ given the rest of stochastic processes. This parameter is a measure of the relative precision of strategic agents' private information about fundamentals at the end of period with respect to their public information. If $\eta$ is very low then we find the lowest level of private signals' precision that is compatible with the uniqueness condition (7). If $\eta$ is very high then private information is much more reliable than public information and signals are so precise about $v_{t}$ that there are low information disparities across traders.

\subsection{Market (Il)liquidity}

In Appendix $\mathrm{A}$ we derive the relationship between $c_{t}^{+}$and $c_{t}^{-}$and a linear residual demand provided by nonstrategic agents. In that context, market illiquidity on the buy and sell side, $c_{t}^{+}$and $c_{t}^{-}$, is linked to market illiquidity and liquidity shocks, $c_{t}$ and $e_{t}$. The former is inversly related to the slope of the residual demand with respect to price, while the latter represents its inelastic component.

Several measures of liquidity have been proposed, (see e.g. Daníelsson and Payne, 2002; Amihud et al., 2005; Pastor and Stambaugh, 2003), some of which depend on proprietary data. In our case, we limit our attention to publicly available data at the daily frequency, whereby the specific market structure dictates the specific form of the model and choice of variables. For example, if the underlying market structure is composed by market makers as on the NYSE, it would be natural to use TAQ data. Alternatively, for limit order markets one might use order flow if available.

The market illiquidity process $c_{t}$ is linked to the uncertainty about the realization of the (discounted value of) future dividends $x_{t}$ around asset fundamentals $f_{t}$, which represents their expected value. If we think of $x_{t} \sim N\left(f_{t}, \sigma_{t}^{2}\right)$ then $\sigma_{t}^{2}$ is the corresponding conditional variance and we expect a positive link between $\sigma_{t}^{2}$ and $c_{t}$. Since $\sigma_{t}^{2}$ is not an observable variable, it can be proxied (e.g. by global volatility) or treated as a latent process.

In addition, the process $c_{t}$ is also determined by how many units of the asset, say $k_{t} \geq 0$, each strategic agent trades in each time period, where up to now we have assumed that $k_{t}=1$. The higher $k_{t}$ is, the riskier is to absorb orders for nonstrategic agents and the higher the market illiquidity $c_{t}$. Similarly to $\sigma_{t}^{2}, k_{t}$ is a latent variable, but unfortunately without the availability of a 
good proxy. Consequently we model its dynamics as negatively related to fundamental uncertainty $\alpha_{t}^{-1}$. We interpret this relationship as external risk controls and asset allocation constraints on strategic traders choices that are based on public information.

There are liquidity tensions (the market is not infinitely deep) if a linear function of both the leverage and uncertainty proxies exceeds a certain threshold, which without loss of generality, we can set at zero. Consequently, we model the illiquidity index $c_{t}$ as a truncation of a linear function of both the leverage and uncertainty proxies

$$
c_{t}=\max \left\{\phi_{0}+\phi_{1} z_{t}^{\mathrm{L}}+\phi_{2} z_{t}^{\mathrm{U}}, 0\right\},
$$

where the proxies are scaled to have mean zero and variance one and denoted by $z_{t}^{\mathrm{L}}$ and $z_{t}^{\mathrm{U}}$ respectively. ${ }^{6}$ In this case $\phi_{0}$ gives the average value of the latent process underlying $c_{t}$, with both the leverage and uncertainty parameters $\left(\phi_{1}, \phi_{2}\right)$ being positive. The relative magnitude of these two parameters indicates the relative importance of leverage versus uncertainty in the determination of market liquidity.

Finally, the price impacts $c_{t}^{-}$and $c_{t}^{+}$are also affected by liquidity shocks $e_{t}$ of nonstrategic traders, which introduce a wedge between $c_{t}^{-}$and $c_{t}^{+}$. The potential asymmetry between $c_{t}^{-}$and $c_{t}^{+}$is succinctly captured by making one of $c_{t}^{+}$or $c_{t}^{-}$, say $c_{t}^{+}$, equal to $c_{t}$ and modelling directly the asymmetry of the other price impact:

$$
c_{t}^{+}=c_{t},
$$

while the asymmetry between $c_{t}^{-}$and $c_{t}^{+}$is given by the following transformation

$$
c_{t}^{-}=c_{t}^{\xi}, \quad \xi \geq 0
$$

where $\xi$ gives a measure of the asymmetry on the buy and sell side of the market. Both $c_{t}^{+}$and $c_{t}^{-}$increase with $c_{t}$, but the mapping (12) captures three different scenarios of the relationship between $c_{t}$ and $e_{t}$ depending on the value of $\xi$ :

$\xi>1 \quad c_{t}^{+}$increases at a constant rate with $c_{t}$ while $c_{t}^{-}$increases at an increasing rate, the former being higher than the latter when $c_{t}<1$ and

\footnotetext{
${ }^{6}$ Specifically, we define

$$
z_{t}^{\mathrm{L}} \equiv-\left(-\log \alpha_{t}-\gamma_{1}\right) / \sqrt{\gamma_{3}^{2} /\left(1-\gamma_{2}^{2}\right)},
$$$$
z_{t}^{\mathrm{U}} \equiv\left(\log \sigma_{t}^{2}-\mathrm{E}\left(\log \sigma_{t}^{2}\right)\right) / \sqrt{\operatorname{Var}\left(\log \sigma_{t}^{2}\right)} .
$$ 
lower when $c_{t}>1$, with the gap increasing in $c_{t}$. As $c_{t}$ increases, this scenario represents an inelastic demand $e_{t}<0$ of nonstrategic traders that changes to an inelastic supply $e_{t}>0$ at level $c_{t}=1$, that is, a positive relationship between $c_{t}$ and $e_{t}$. Moreover, we can interpret the increasing gap between price impacts (final inelastic supply is higher than initial inelastic demand) as the unwinding of accumulated positions.

$\xi<1 c_{t}^{-}$increases at a decreasing rate, being higher than $c_{t}^{+}$when $c_{t}<1$ and lower when $c_{t}>1$, with the gap increasing in $c_{t}$. As $c_{t}$ increases, this scenario represents an inelastic supply of nonstrategic traders that changes to an inelastic demand at level $c_{t}=1$, that is, a negative relationship between $c_{t}$ and $e_{t}$.

$\xi=1 c_{t}^{-}=c_{t}^{+}$and both increase at a constant rate with $c_{t}$. This scenario represents the lack of an inelastic component $e_{t}$ in the residual demand of nonstrategic traders.

\section{Estimation Method}

Econometrically, our model is a special form of a stochastic volatility (SV) model. Due to the nonlinear dependence on nonnormal dynamic latent variables such models cannot be directly estimated by either maximum likelihood or GMM. However, simulation methods have successfully been applied to SV models and we follow that approach here, in particular the efficient method of moments (EMM), proposed by Gallant and Tauchen $(1996,2002)$. The deep parameters of our model are

$$
\rho=\left(\gamma_{0}, \gamma_{1}, \gamma_{2}, \gamma_{3}, \eta, \phi_{0}, \phi_{1}, \phi_{2}, \xi\right)
$$

Denote by $\mathbf{x}_{t}$ a vector of lagged returns plus strictly exogenous covariates if they are introduced in the model to proxy $m_{t}$ and $z_{t}^{\mathrm{U}}$. Our model defines implicitely a particular dynamic density $p\left(r_{t} \mid \mathbf{x}_{t} ; \rho\right)$ for given deep parameters $\rho$.

\subsection{First EMM Step: SNP Estimation}

The EMM approach is based on first estimating an auxiliary model of the observable data, the score generator, with the SNP semi nonparametric method. The SNP density is based on the notion that a Hermite expansion can be 
used to provide a general purpose approximation of the density function of the data of interest, with parametric modelling capturing dependence in the first and second moments. The SNP method nests many variants of ARGARCH models.

While our theoretical model only defines an implicit density, this reduced form model has an explicit density that we can define as $f\left(r_{t} \mid \mathbf{x}_{t} ; \theta\right)$, where $\theta$ is the corresponding parameter vector. The semi nonparametric feature is due to the dimension of $\theta$, specifically the Hermite expansion order, which should be expanded at a proper rate as $T$ grows to get the same efficiency as maximum likelihood when applying SNP to EMM.

Take the dimension of $\theta$ as given. While we don't know the true parameters $\theta_{0}$ of the score generator, we can estimate them by quasi-maximum likelihood. That is, the estimator of $\theta_{0}$ is a $\hat{\theta}$ such that $\sum_{t=1}^{T} \mathbf{s}\left(r_{t} \mid \mathbf{x}_{t} ; \hat{\theta}\right)=\mathbf{0}$, where $\mathbf{s}(\cdot)$ is the score of $f(\cdot)$

$$
\mathbf{s}\left(r_{t} \mid \mathbf{x}_{t} ; \theta\right)=\frac{\partial}{\partial \theta} \ln f\left(r_{t} \mid \mathbf{x}_{t} ; \theta\right) .
$$

This estimation is done for a given dimension of $\theta$, but we need to pin down that dimension too. Hence this first EMM step also relies on a model selection procedure, such as the Schwarz criteria (BIC). Several SNP models are estimated by quasi-maximum likelihood and the one with lowest BIC is chosen.

\subsection{Second EMM Step: Deep Parameters Estimation}

The score of the chosen SNP model is used to identify the efficient moments to use in the estimation of deep parameters. We know by quasi-maximum likelihood arguments that there is a $\theta_{0}$ related to the true deep parameters $\rho_{0}$ by implicit equations $\mathbf{m}\left(\rho_{0}, \theta_{0}\right)=\mathbf{0}$, where

$$
\mathbf{m}(\rho, \theta)=E_{\rho}\left[\mathbf{s}\left(r_{t} \mid \mathbf{x}_{t} ; \theta\right)\right]
$$

is the expected SNP score with respect to the theoretical model evaluated at $\rho$. Our inference will be based on inverting that relationship.

In the final EMM step, the estimator $\hat{\rho}$ of $\rho_{0}$ minimizes the distance between expected SNP score evaluated at $\hat{\theta}$ and zero for a particular metric,

$$
\hat{\rho}=\arg \min _{\rho} \mathbf{m}(\rho, \hat{\theta})^{\prime} \hat{\mathbf{S}}^{-1} \mathbf{m}(\rho, \hat{\theta})
$$


where $\hat{\mathbf{S}}$ is a consistent estimator of $\mathbf{S}$, the asymptotic variance of $T^{-1 / 2} \sum_{t=1}^{T}$ $\mathbf{s}\left(r_{t} \mid \mathbf{x}_{t} ; \theta_{0}\right)$. Gallant and Tauchen (1996) show sevaral ways of computing $\hat{\mathbf{S}}$ and develop the asymptotic distribution of $\hat{\rho}$.

(13) refers to a model without covariates. However, the EMM methods allows for the inclusion of exogenous explanatory variables in the theoretical model. See Gallant and Tauchen (1996) for the required extensions in the definition of $\mathbf{m}(\cdot)$ and $\mathbf{S}$.

If the score generator smoothly embeds the true model this estimator is fully efficient with the same distribution as the maximum likelihood estimator of $\rho_{0}$. We can expect EMM to be nearly fully efficient when SNP is used as the score generator since it is a flexible reduced form model that closely approximates the actual distribution of the data.

Moreover, given this property of the SNP model, the EMM procedure selects the optimal testable properties of the model. The value of the criterion function provides a good test of the adequacy of a theoretical model to explain the dynamic and steady state properties of data. We can also perform a more detailed analysis moment by moment by means of score diagnostics.

The specific procedures, and in particular the optimization method, MCMC, are described in Appendix B.

\section{Empirical Application}

The relevance of our model ultimately rests on its ability to capture important tumultuous events in financial markets, e.g. speculative attacks which were the original motivation behind Morris and Shin (1998), asset price bubbles, emerging markets crisis, or carry trades. Figure 1 depicts some possible scenarios of market instability which can be captured by our model. Our application is based on carry trades driving the first scenario.

Carry trades are a common trading strategy, whereby market participants borrow funds in a country with low interest rates and invest them in a country with high interest rates. Denote by $\mathrm{FX}^{\mathrm{L}}$ and $\mathrm{FX}^{\mathrm{H}}$ the low and high interest rate country exchange rates respectively. If a sufficient number of agents

follow the strategy it will weaken $\mathrm{FX}^{\mathrm{L}}$, further increasing profits, implying such a strategy is self reinforcing.

However, such a situation is unstable because a small change in the economic environment, say adverse changes in the interest rate differential and/or the exchange rates, can potentially trigger events such as margin calls and high losses in highly leveraged positions. Consequently, agents will be on a con- 
stant lookout for such scenarios and rapidly unwind their trades if they suspect it is imminent. While such trading strategies are sometimes likened to collecting crumbs in front of a steamroller, they nevertheless generally contain significant amount of funds and are a important concern for policy makers.

The slow buildup and the subsequent rapid unwinding of carry trades implies that exchange rates follow what has been called the up by the escalator-down by the elevator pattern for exchange rates. This scenario translates to our model as $\xi>1$, that is, a positive relationship between nonstrategic agents' liquidity shocks $e_{t}$ and market illiquidity $c_{t}$. We take $\mathrm{FX}^{\mathrm{L}}$ as the numeraire in the following.

Consider a context of moderate illiquidity $c_{t}$. There is a natural initial direction of the nonstrategic agents' inelastic demand in this case due to the interest rate differential, buy $\mathrm{FX}^{\mathrm{H}}$ and sell $\mathrm{FX}^{\mathrm{L}},\left(e_{t}<0\right)$, making the buy side relatively more illiquid $\left(c_{t}^{-}<c_{t}^{+}\right)$and hence giving strategic agents incentives to buy $\mathrm{FX}^{\mathrm{H}}$ also. Many strategic traders will buy $\mathrm{FX}^{\mathrm{H}}$ unless the realization of $v_{t}$ is quite low.

However, a context of increasing leverage and/or uncertainty translates into increasing market illiquidity $c_{t}$. The positions of nonstrategic agents in $\mathrm{FX}^{\mathrm{H}}$ tend to be unwound when $c_{t}$ passes a critical level, that is, their inelastic demand becomes sell $\mathrm{FX}^{\mathrm{H}}$ and buy $\mathrm{FX}^{\mathrm{L}}\left(e_{t}>>0\right)$. This makes the sell side much more illiquid than the buy side $\left(c_{t}^{-}>>c_{t}^{+}\right)$which implies that strategic agents have strong incentives to sell $\mathrm{FX}^{\mathrm{H}}$ too. Eventually, even a relatively high realization of $v_{t}$ might not avoid that many strategic traders sell, which leads to a rapid depreciation in $\mathrm{FX}^{\mathrm{H}}$.

\subsection{The Yen-Dollar Crisis of 1998}

One of the best known examples of a carry trade induced market turmoil occured in 1998, when over two days in October $-7^{\text {th }}$ and $8^{\text {th }}$ - the dollar fell from 131 yen to 112 yen by lunchtime in London on Thursday the $8^{\text {th }}$, bouncing back sharply to end New York trading at 119 yen. October $7^{\text {th }}$ and $8^{\text {th }} 1998$ were two of the most turbulent days of currency trading in financial markets in recent memory. These events are discussed e.g. by Morris and Shin (2000) and Daníelsson and Shin (2003). In the week beginning October $5^{\text {th }}$, the decline of the dollar against the yen accelerated sharply - closing down roughly $15 \%$ over the week consistent with the rapid unwinding of the yen carry trades. Global events such as the Russian default, LTCM, and the yield curve adjustments leading to the Euro initiated the unwinding of 
previous long positions in dollars.

We use daily yen dollar exchange rates as reported by the New York Fed and estimate the model using continuously compounded returns in percentage terms, $r_{t}$. While our main event of interest occurs in 1998, the entire data sample used for estimation spans 1992 to 2004. Since our model addresses both liquid and illiquid time periods, the data need to be sufficiently long so that we obtain a good estimation of the underlying processes of fundamental and strategic returns. At the same time we avoid an arbitrary choice of time period to be fitted by the model.

We also use proxies of fundamentals and payoff uncertainty. The fundamental mean $m_{t}$ is driven by the interest rate spread between the US and Japan, since it can be interpreted as the dividend of holding dollars and it is available at the daily frequency. ${ }^{7}$ Indicate $i^{\mathrm{JAP}}$ and $i^{\mathrm{US}}$ the daily rate of return on the monthly ${ }^{8}$ LIBOR Japanese and US interest rates, respectively. This data was obtained directly from www.bba.org. The interest rate spread is calculated by $s_{t-1}=100 \log \left[\left(1+i_{t-1}^{\mathrm{JAP}}\right) /\left(1+i_{t-1}^{\mathrm{US}}\right)\right]$ and the fundamental mean is linearly related to the spread's first difference $z_{t-1}^{\mathrm{F}}=\Delta s_{t-1}$. Therefore, the sign of $\gamma_{0}$ is informative about the connection between interest rate news and fundamental appreciation. ${ }^{9}$ The payoff uncertainty $\sigma_{t}^{2}$ is proxied by the volatility in global markets, in particular the MSCI index denominated in US dollars. If the daily log return on the index is $y_{t}$ in percentage terms then the volatility forecast that we use is simply $\sigma_{t}^{2}=y_{t-1}^{2}$, similar to ARCH filtering of volatility.

Summary statistics for the data are shown in Table 1, and plots of both exchange rates and returns in Figures 2-3. The MSCI returns and interest rate spreads are shown in Figures 4 and 5 respectively.

We identify three general regimes within our sample period by considering descriptive accounts of the sample period and statistical analysis of the data. Most importantly, our key event occurs in 1998, with the escalator effects commencing in 1996 and the aftershocks terminating in 1999. This can also be identified from the interest rate spreads which are lowest in the same time

\footnotetext{
${ }^{7}$ Other macro variables such as real output and nominal money supply are not available at the daily frequency.

${ }^{8} \mathrm{We}$ should use overnight rates but the corresponding time series (2001-2005) is not long enough. Fortunately, the behaviour of overnight rates is closely related to monthly rates. For the period of time were both are available, the correlation is 0.975 and OLS of overnight rates onto one-month rates gives an intercept of -0.001 and a slope of 0.964 .

${ }^{9}$ The fundamental return parameters are related to the arrival of new information in the economic model and hence we use $\Delta s_{t-1}$ instead of $s_{t-1}$. In the empirical application, the use of $\Delta s_{t-1}$ instead of $s_{t-1}$ does not have a significant impact on the results.
} 
period. Consequently, the second subsample spans 1996 to 1999. Starting around 1992 and ending in 1995, we see the interest rate spreads decline systematically, along with the exchange rates. Also, the MSCI index has relatively low volatility in those years. Finally, after 1999 the spreads are increasing, MSCI volatility is high, and the exchange rates are relatively stable. Taken together, this suggests estimating our models over three subsamples in addition to the entire sample, i.e., 1992-1995, 1996-1999, and 2000-2004.

We followed the procedure in Gallant and Tauchen (2006) to select the SNP densities for both the full sample and the subsamples, which results in model 11114010 for the full sample and 11114000 for each subsample. Since 11114010 has 14 parameters while 11114000 only 9, we suspect the larger sample size for the full sample implied the richer model. Both SNP models are $\mathrm{AR}(1)-\mathrm{GARCH}(1,1)$ with a semiparametric density for innovations based on a fourth order Hermite polynomial. We used hundred thousand simulations in EMM.

\subsection{Model Estimation}

We estimate three variants of our model:

Model $A$ is a standard stochastic volatility (SV) model, which is represented by an infinitely deep market in our framework. We also set the fundamental mean $m_{t}$ to zero and hence there are only three deep parameters, $\rho_{A}=\left(\gamma_{1}, \gamma_{2}, \gamma_{3}\right)$.

Model $B$ introduces potential liquidity tensions using only information of FX returns. This is achieved by setting the fundamental mean $m_{t}$ to zero and assuming payoff uncertainty $z_{t}^{\mathrm{U}}$ is IID normal. This results in parameters, $\rho_{B}=\left(\gamma_{1}, \gamma_{2}, \gamma_{3}, \eta, \phi_{0}, \phi_{1}, \phi_{2}, \xi\right)$.

Model $C$ incorporates both changes in the interest rate spreads and the volatility of the MSCI index as proxies ${ }^{10}$ for fundamentals and payoff uncertainty, implying parameters, $\rho_{C}=\left(\gamma_{0}, \gamma_{1}, \gamma_{2}, \gamma_{3}, \eta, \phi_{0}, \phi_{1}, \phi_{2}, \xi\right)$.

Table 2 presents the parameter estimates results which describe the environment of fundamentals and liquidity under each model. In all cases the parameters for the full sample are statistically different from zero (the only

\footnotetext{
${ }^{10}$ The proxies are treated as strictly exogenous and stationary covariates in the implementation of the model. Exogeneity was checked with empirical tests based on VAR(1)s. The lagged FX return did not help to forecast these proxies with respect to their own lag.
} 
exception being $\gamma_{3}$ in Model $B$ ), with $\xi$ also significantly different from one, but some of the subsample parameters are not, indicating the subsample size may not be sufficient. We initially estimated $\eta$ along with the other model parameters, but in all cases the results indicated unbounded signals precision $\beta_{t} \rightarrow \infty$. That is, low information disparities across strategic traders, which is a natural feature of FX markets. Consequently, the estimates in the table refer to models where $\beta_{t} \rightarrow \infty$.

In the case of Model $A$, the estimated parameters are $(-1.28,0.61,0.56)$, with a somewhat low persistence parameter. A likely reason is that the presence of outliers or excessive market turmoil hides the volatility clusters resulting in a low estimation of the persistence parameter. Similar results obtain in the subsamples, where the second subsample shows the highest $\hat{\gamma}_{1}$ and $\hat{\gamma}_{2}$ to fit liquidity tensions. Model $A$ is rejected by the data at all periods given the high criterion function's values.

The criterion function value for Model $B$ implies that it is not rejected by the data. This model shows a lower SV of fundamental returns than Model $A$ on average, $\hat{\gamma}_{1}=-1.87$, and considerable degree of persistence with $\hat{\gamma}_{2}=0.99$.

The asymmetry parameter $\hat{\xi}=1.38$ is consistent with the up by escalatordown by elevator scenario. For the market illiquidity process, we find that $\hat{\phi}_{0}=-0.39$ which indicates that strategic agents only affect the market at certain times since the market is usually infinitely deep. The leverage parameter $\hat{\phi}_{1}=0.48$ indicates that fundamental volatility is negatively correlated with market illiquidity, i.e. as strategic agents decreasing leverage under increasing fundamental volatility. The random proxy for payoff uncertainty is associated to $\hat{\phi}_{2}=0.79$, and this value shows that this factor is relatively more important than fundamental uncertainty.

Results for the subperiods indicate that the fundamental parameters are stable across the periods while the liquidity parameters are not. However, across all periods we find $\hat{\phi}_{0}<0$ and $\hat{\xi}>1$. The second subperiod, which contains many crisis events, has the highest $\hat{\xi}$, i.e. that is, the maximum asymmetry on the sell side, as well as the highest $\hat{\phi}_{0}$.

Finally, Model $C$, introduces the change in interest rate spreads with parameter $\gamma_{0}$ and global volatility with parameter $\phi_{2}$. This model is not rejected by EMM either. We find $\hat{\gamma}_{0}=-0.12$, a fundamental appreciation of the dollar is expected when the spread between Japan and USA interest rates decreases. ${ }^{11}$ On the other hand, the SV component of fundamental returns

\footnotetext{
${ }^{11}$ An uncovered interest rate parity (UIP ) argument links a negative spread with a dollar (observed) depreciation. It is well known that UIP is at odds with empirical evidence. See for instance Evans and Lyons (2002).
} 
shows a higher average level than Model $B$ with $\hat{\gamma}_{1}=-1.74$, and lower persistence with $\hat{\gamma}_{2}=0.48$, a crowding-out effect of global market volatility through market illiquidity.

As in Model $B$, the asymmetry parameter $\xi$ is consistent with the escalatorelevator scenario with $\hat{\xi}=1.53$, the market are usually very deep since the estimation of average illiquidity is $\hat{\phi}_{0}=-1.13$, and the uncertainty parameter is higher than the leverage one with $\hat{\phi}_{1}=0.22$ and $\hat{\phi}_{2}=1.61$. The latter indicates that high global market volatility, as a proxy for payoff uncertainty, is related to low liquidity for the yen carry trades. Furthermore, global volatility is more important than fundamental uncertainty in the determination of liquidity.

Subsamples also show a negative (but nonsignificant) $\hat{\gamma}_{0}$. We do find significant differences in the SV model between subsamples, in particular $\hat{\gamma}_{2}$. By contrast, $\hat{\gamma}_{2}$ is high in all samples for Model $B$, suggesting that the change in magnitudes is entirely due to the covariates. The discussion of the liquidity parameters $\hat{\phi}_{0}$ and $\hat{\xi}$ in Model $B$ applies here too.

In sum, the results clearly indicate that a standard SV model is not suitable to explain the dollar exchange rate, while our model is. Furthermore, the introduction of covariates significantly affects the results, and provides clear identification of different environments of fundamentals and liquidity.

\subsection{Model Simulation}

We further illustrate the estimation results by means of simulations whereby we simulate the three models over all subsamples with parameter estimates from Table 2. Since this results in extensive output, we only present representative results here. The full Monte Carlo results are available from us upon request. In all cases the number of simulations is 100,000, while for the figures the simulation size is 1000 . First, we study more the liquidity context that our model is describing. Second, we study the properties of returns and prices under our model estimates.

For Model $B$, Table 3 focuses on the relative magnitude of $c^{+}$and $c^{-}$during the second subsample where the market turmoil was high. The Table shows the relative frequency and magnitude of $c^{+}, c^{-}>i \times \sigma$ (yen returns), $i=$ $0,1, \ldots, 5$, i.e., the speculative price impacts above thresholds of increasing size as multiples of the volatility of the yen dollar exchange rate returns. The asymmetry between the buy and sell sides is clear, in particular, for the largest thresholds $c^{+}$is zero but not for $c^{-}$. This has direct implications for asset price dynamics and results in the elevator - escalator effect. 
For Model $C$, Table 4 shows sample statistics for the observed data, the fundamental return $v$ and the simulated total return, $r$. We see that the statistics of $v$ do not match the observed yen returns while the total returns do. Especially interesting, the kurtosis, minima, maxima and autocorrelations are similar. This suggests that the model has successfully captured the stylized statistical facts of the yen dollar exchange rate.

Finally, Figures 6 and 7 show the first thousand simulated exchange rates for the full sample and the second subsample, respectively. Here, especially in the second figure, the elevator - escalator effects are very dramatic.

\section{Conclusions and Further Research}

This paper represents a first attempt to the integration of coordination games with heterogeneous information and empirical finance, specifically stochastic volatility models. There are some interesting issues, both theoretical and empirical, that are outside the scope of this paper, and hence are left for future research.

It is possible to filter and forecast the latent variables, such as the fundamental and strategic components of returns which is helpful for the identification of unstable periods and the potential for liquidity tensions, and as such could be of use to both policymakers and practitioners. While such filtering and forecasting is conceptually straightforward, it is technically somewhat challenging and beyond the scope of this paper. Another avenue of empirical extensions would be the use of richer data in the estimation, e.g. by making better use of the microstructure of the underlying markets.

It is also be of interest to specify agent behavior in a more detailed manner, e.g. to understand better the liquidity provided by nonstrategic agents by building a more primitive model of the residual demand they represent and hence estimating deeper parameters. On the other hand, it would be interesting to enrich the information structure and dynamic behavior of strategic agents. The informational content of prices in global games has recently been studied by Angeletos and Werning (2005), Hellwig et al. (2006), and Tarashev (2003). Similarly, richer dynamic behavior in global games (e.g. learning across several trading rounds) is considered ${ }^{12}$ by Angeletos et al. (2006), and Dasgupta (2007).

\footnotetext{
${ }^{12}$ Abreu and Brunnermeier (2003) is another relevant reference on dynamic behavior, close in spirit to global games.
} 


\section{A Return Decomposition}

We represent nonstrategic trades by means of a linear residual demand, which defines the environment of fundamentals and liquidity that strategic traders face and might be interpreted as a limit order book,

$$
\frac{f-p}{d}-e,
$$

where $f$ is the expected liquidation value of the asset under study, termed the asset fundamentals, $d$ is a nonnegative parameter, and $e$ is a parameter that takes values on the real line.

This net demand schedule can be easily motivated by a representative competitive mean-variance agent. Suppose this trader has only access to two assets. The first a risky asset with price $p$ and random payoff, or liquidation value, $x \sim N\left(f, \sigma^{2}\right)$. The second asset is riskless with price normalized to 1 and rate of return fixed to 0 , without loss of generality. ${ }^{13}$ If we further assume that she maximizes a CARA utility defined on final wealth with a coefficient of absolute risk aversion $a$ then her demand of the risky asset is $(f-p) / d$ with $d=a \sigma^{2}$.

The second component $e$ of the net demand can be interpreted as an endowment of the risky asset or a liquidity shock realized at the beginning of the period. Hence $e>0$ means that there is some inelastic supply of the risky asset from the representative nonstrategic trader, while $e<0$ means that there is some inelastic demand of the risky asset. Given that the measure of strategic traders is 1 , we assume $|e| \leq 1$ to model their relevance in this market.

On the other hand, the (per capita) net supply of strategic traders is $\lambda-$ $(1-\lambda)$ since they can only sell or buy one asset unit each. The closing price, the price by the end of trading period, will be given by the market clearing condition of the risky asset. The equality of net demand by nonstrategic traders and net supply by strategic traders by the end of trading period expressed as

$$
\frac{f-p}{d}-e=2 \lambda-1
$$

gives the equilibrium price

$$
p=f-d e-d(2 \lambda-1)=f+d(1-e)(1-\lambda)-d(1+e) \lambda .
$$

\footnotetext{
${ }^{13}$ Moreover, it is not far from reality if the trading period is one day.
} 
Denote the previous closing price by $p_{0}$. The current closing price $p$ translates into the following rate of return for the risky asset:

$$
r=\frac{p-p_{0}}{p_{0}}=v-\lambda c^{-}+(1-\lambda) c^{+}
$$

where we have defined

$$
v=\frac{f-p_{0}}{p_{0}}, \quad c^{-}=c(1+e), \quad c^{+}=c(1-e), \quad c=\frac{d}{p_{0}},
$$

and both $\left(c^{+}, c^{-}\right)$are nonnegative given our assumption on $e$.

If the market is infinitely deep, $c=0$, then prices are equal to fundamentals and $r=v$. If there is not an inelastic component in the residual demand, $e=0$, then $c^{+}=c^{-}=c$, that is, both directions of strategic traders orders are equally absorbed and give the same deviation of $p$ with respect to $f$ and hence $r=v-c(2 \lambda-1)$.

Naturally, $e>0$ implies $c^{-}>c^{+}$whenever $c \neq 0$. That is, if there is some inelastic supply (a positive liquidity shock) is is easier for the market to absorb buying orders from strategic traders, in the sense that strategic buying requires a smaller deviation of $p$ with respect to $f$ than strategic selling. Similarly, if $e<0$ then $c^{+}>c^{-}$, some inelastic demand (a negative liquidity shock) implies a market that absorbs easier selling orders from strategic traders.

Finally, we can extend the model to a set-up where strategic traders can trade a number of asset units $k$ not necessarilly equal to 1 . To simplify, let us assume that the liquidity shock is also scaled by $k$. Then the market clearing condition becomes

$$
\frac{f-p}{d}-k e=k(2 \lambda-1)
$$

and we can see that the effect of $k$ is equivalent to scaling $d$ by $k$, and hence scaling $c$.

\section{B Simulated Scores and MCMC}

We use two different types of simulations in the second step of EMM. First, while we cannot compute $\mathbf{m}(\rho, \theta)$ analytically, it is straightforward to get a good approximation by means of simulations. Specifically, $\mathbf{m}(\rho, \theta)$ is an expectation and can be approximated by a simulated sample average. We 
can simulate a long time series $\left\{r_{h}(\rho)\right\}_{h=1}^{H}$ from $p\left(r_{t} \mid \mathbf{x}_{t} ; \rho\right)$ and use the approximation:

$$
\mathbf{m}(\rho, \theta) \simeq \frac{1}{H} \sum_{h=1}^{H} \mathbf{s}\left(r_{t}(\rho) \mid \mathbf{x}_{t}(\rho) ; \theta\right)
$$

The theoretical model is simulated for given model parameters $\rho$ and the SNP scores evaluated at $\hat{\theta}$ are calculated from simulated data. Finally, the estimation of deep parameters is based on making the average of simulated SNP scores close to zero. If $H$ if sufficiently large, the sampling error in (14) is negligible. In the case where we use exogenous variables, were generally their sample size $T$ is much lower than $H$, we employ repeated sequences of the exogenous variables. We can proceed with the calculation in (14) provided exogenous variables are strictly stationary and exogenous.

We obtain parameters estimates by the Markov chain Monte Carlo (MCMC) approach of Chernozhukov and Hong (2003), where deep parameter estimates are computed by a quasi-Bayesian MCMC method. In our case, the quasi-likelihood for $\rho$ is $\exp \left[-T \mathbf{m}(\rho, \hat{\theta})^{\prime} \hat{\mathbf{S}}^{-1} \mathbf{m}(\rho, \hat{\theta})\right]$ and can be used to apply Bayesian MCMC methods to estimate $\rho_{0}$. Specifically, we will use a Metropolis-Hastings sampler based on a random walk and a normal proposal.

The advantage of using and MCMC procedure is the convenience of imposing support restrictions, inequality restrictions, and informative but not sharp priors. These restrictions and priors can be imposed on the model parameters or arbitrary functions of the model. For example, instead of the procedure wasting time generating data that is clearly at odds with the true data, we can expediently reject those parameter estimates with that rejection learned. Finally, the MCMC output provides a natural way to obtain standard errors of the EMM estimators. 


\section{Tables and Figures}

Table 1: Summary statistics daily yen-dollar exchange rate returns and covariates, 1992-2004

The yen-dollar and MSCI index are in percentage returns, same as in Figures 3, and 4, respectively. The spread is the Japanese one month LIBOR minus the US one month LIBOR, same as in Figure 5.

\begin{tabular}{|c|c|c|c|}
\hline Statistic & yen-usd & MSCI index & spread \\
\hline Mean: & -0.01 & 0.02 & -0.03 \\
\hline Std & 0.70 & 0.85 & 0.02 \\
\hline Skewness: & -0.50 & -0.12 & 0.18 \\
\hline Kurtosis: & 3.98 & 3.25 & -1.31 \\
\hline Min: & -5.63 & -5.21 & -0.06 \\
\hline Max: & 3.24 & 4.79 & 0.02 \\
\hline
\end{tabular}


Table 2: Estimation results

Standard errors in parenthesis. Estimates with unbounded signals precision, i.e. $\beta_{t} \rightarrow \infty$.

\begin{tabular}{lrrrrrrrr}
\hline model & $\gamma_{0}$ & $\gamma_{1}$ & $\gamma_{2}$ & $\gamma_{3}$ & $\phi_{0}$ & $\phi_{1}$ & $\phi_{2}$ & $\xi$ \\
\hline A & & -1.28 & 0.607 & 0.555 & & & & \\
full sample & $(0.063)$ & $(0.045)$ & $(0.042)$ & & & & \\
\hline A & & -1.41 & 0.435 & 0.783 & & & & \\
$92-95$ & & $(0.080)$ & $(0.655)$ & $(0.063)$ & & & & \\
\hline A & & -0.926 & 0.69 & 0.435 & & & & \\
$96-99$ & & $(0.173)$ & $(0.456)$ & $(0.104)$ & & & & \\
\hline A & & -1.22 & 0.577 & 0.539 & & & & \\
2000-04 & & $(0.082)$ & $(0.075)$ & $(0.066)$ & & & & \\
\hline B & & -1.87 & 0.99 & 0.02 & -0.393 & 0.476 & 0.79 & 1.38 \\
full sample & & $(0.079)$ & $(0.001)$ & $(0.017)$ & $(0.047)$ & $(0.038)$ & $(0.018)$ & $(0.035)$ \\
\hline B & & -1.6 & 0.986 & 0.146 & -3.06 & 1.82 & 1.48 & 1.09 \\
$92-95$ & & $(0.093)$ & $(0.002)$ & $(0.034)$ & $(0.371)$ & $(0.066)$ & $(0.132)$ & $(0.064)$ \\
\hline B & & -1.64 & 0.99 & 0.146 & -0.056 & 0.002 & 0.674 & 1.98 \\
$96-99$ & & $(0.211)$ & $(0.003)$ & $(0.047)$ & $(0.12)$ & $(0.179)$ & $(0.09)$ & $(0.207)$ \\
\hline B & & -1.77 & 0.987 & 0.109 & -0.53 & 0.343 & 0.751 & 1.33 \\
2000-04 & & $(0.085)$ & $(0.003)$ & $(0.036)$ & $(0.099)$ & $(0.059)$ & $(0.045)$ & $(0.127)$ \\
\hline C & -0.123 & -1.74 & 0.482 & 0.324 & -1.13 & 0.219 & 1.61 & 1.53 \\
full sample & $(0.034)$ & $(0.033)$ & $(0.113)$ & $(0.091)$ & $(0.017)$ & $(0.038)$ & $(0.016)$ & $(0.018)$ \\
\hline C & -0.01 & -1.47 & 0.988 & 0.126 & -3.89 & 1.91 & 3.35 & 1.1 \\
$92-95$ & $(0.128)$ & $(0.105)$ & $(0.002)$ & $(0.039)$ & $(0.395)$ & $(0.435)$ & $(0.300)$ & $(0.080)$ \\
\hline C & -0.199 & -1.66 & 0.761 & 0.589 & -0.302 & 0.308 & 0.945 & 2.53 \\
$96-99$ & $(0.303)$ & $(0.291)$ & $(0.148)$ & $(0.232)$ & $(0.119)$ & $(0.097)$ & $(0.088)$ & $(0.285)$ \\
\hline C & -0.024 & -1.73 & 0.307 & 0.469 & -1.01 & 0.824 & 0.767 & 1.19 \\
2000-04 & $(0.062)$ & $(0.096)$ & $(0.215)$ & $(0.219)$ & $(0.154)$ & $(0.083)$ & $(0.105)$ & $(0.111)$ \\
\hline & & & & & & & &
\end{tabular}


Table 3: Some simulation results from Model $C$ in subsample 1996-1999

Simulated sample statistics from Model $C$ with parameter estimates from Table 2, and 100,000 simulations. Relative frequency and magnitude of $c^{+}, c^{-}>i \times \sigma$ (yen returns), $i=$ $0,1, \ldots, 5$, i.e., the speculative price impacts above thresholds of increasing size as multiples of the volatility of the yen dollar exchange rate returns.

\begin{tabular}{rrrrrr}
\hline & \multicolumn{2}{c}{$c^{-}$} & & \multicolumn{2}{c}{$c^{+}$} \\
\cline { 2 - 3 } \cline { 5 - 6 } magnitude & frequency & mean & & frequency & mean \\
$0 \times \sigma$ & $17.7 \%$ & 0.64 & & $23.3 \%$ & 0.48 \\
$1 \times \sigma$ & $4.68 \%$ & 1.96 & & $3.4 \%$ & 1.00 \\
$2 \times \sigma$ & $2.28 \%$ & 2.75 & & $0.001 \%$ & 1.67 \\
$3 \times \sigma$ & $1.05 \%$ & 3.6 & & $0 \%$ & - \\
$4 \times \sigma$ & $0.48 \%$ & 4.47 & & $0 \%$ & - \\
$5 \times \sigma$ & $0.23 \%$ & 5.3 & $0 \%$ & - \\
\hline
\end{tabular}

Table 4: Simulation of Model $C$, full sample

Simulated sample statistics from Model $C$ with parameter estimates from Table 2, and 100,000 simulations.

\begin{tabular}{lrrrrrrrr}
\hline mean & data & sd & skew & kurt & $\min$ & $\max$ & $\operatorname{ar}(y)$ & $\operatorname{ar}\left(y^{2}\right)$ \\
\hline yen returns & -0.0097 & 0.703 & -0.504 & 3.97 & -5.63 & 3.24 & 0.025 & 0.212 \\
$v$ & -0.0007 & 0.452 & -0.037 & 0.89 & -3.48 & 3.01 & 0.005 & 0.029 \\
$r$ & -0.0256 & 0.727 & -0.832 & 4.20 & -5.83 & 3.73 & 0.019 & 0.131 \\
\hline
\end{tabular}


Figure 1: Scenarios of market instability

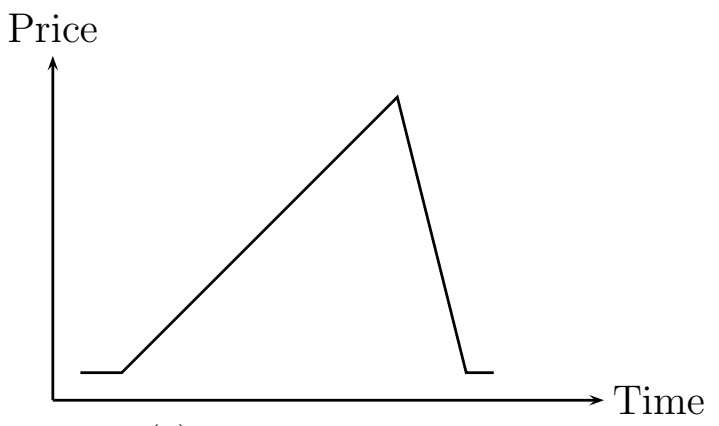

(a) Up by escalator-down by elevator

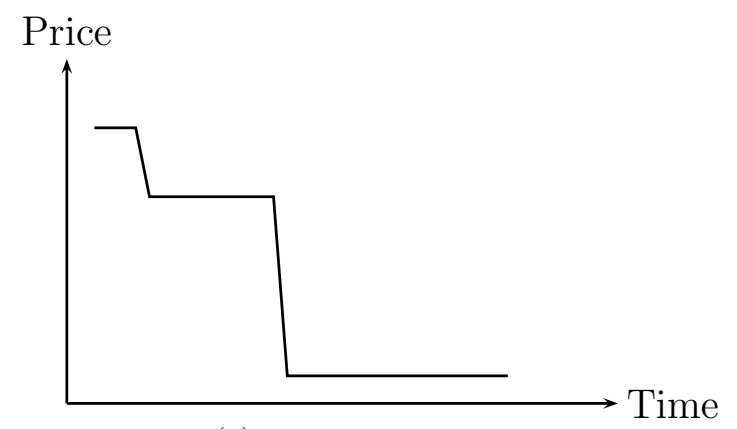

(c) Repeated down by elevator

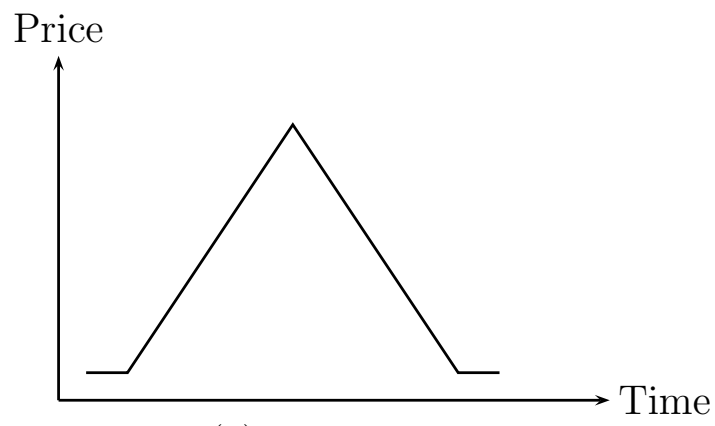

(b) Up and down by escalator

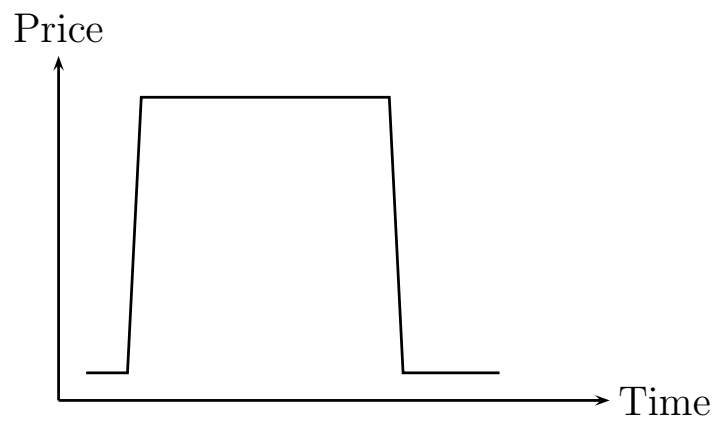

(d) Up and down by elevator

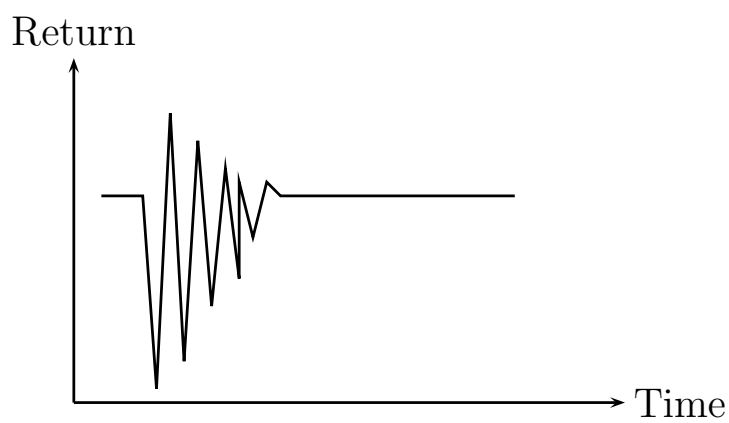

(e) Occasional extreme volatility 
Figure 2: Yen-Dollar 1992-2004
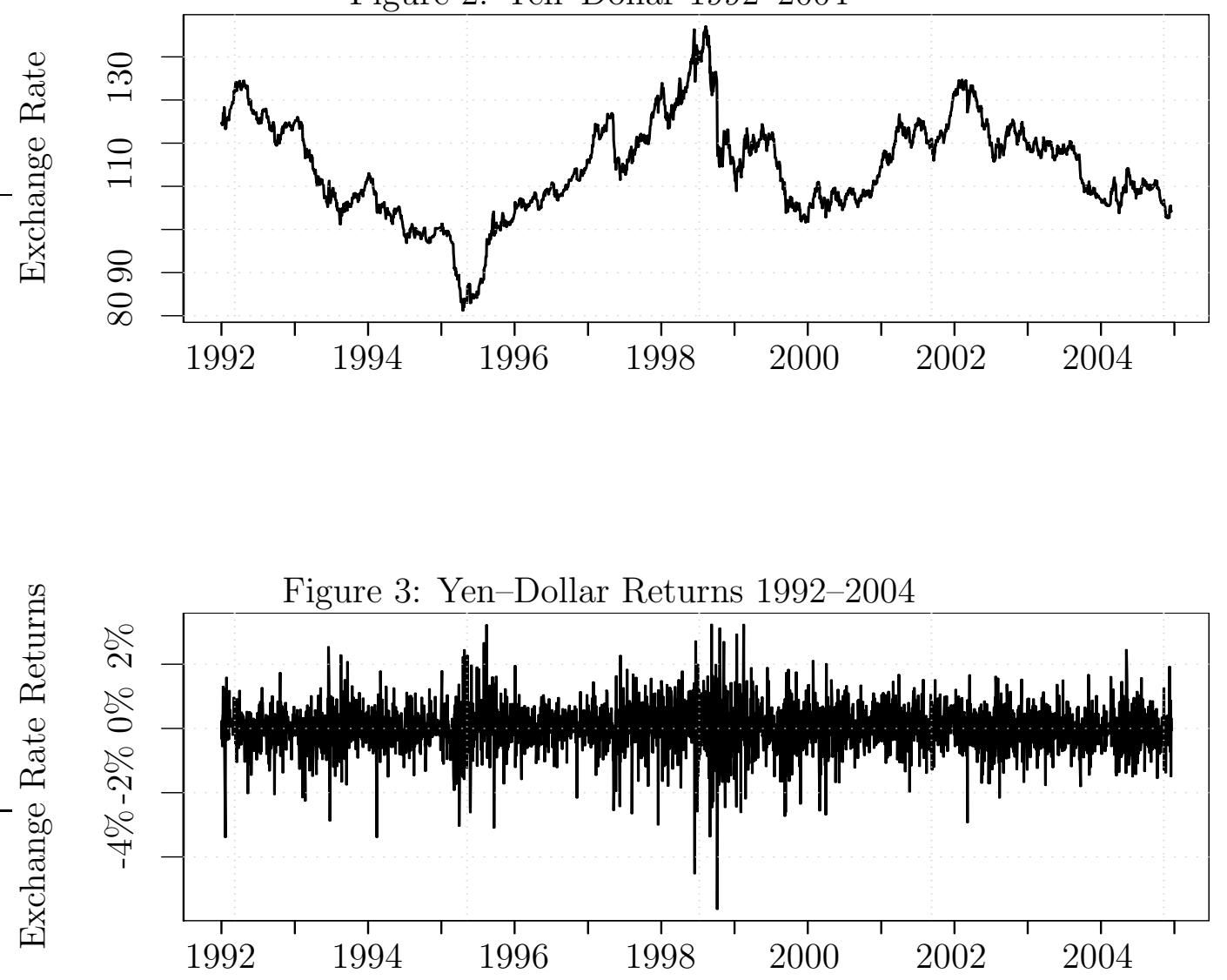

Figure 4: MSCI Index Returns 1992-2004

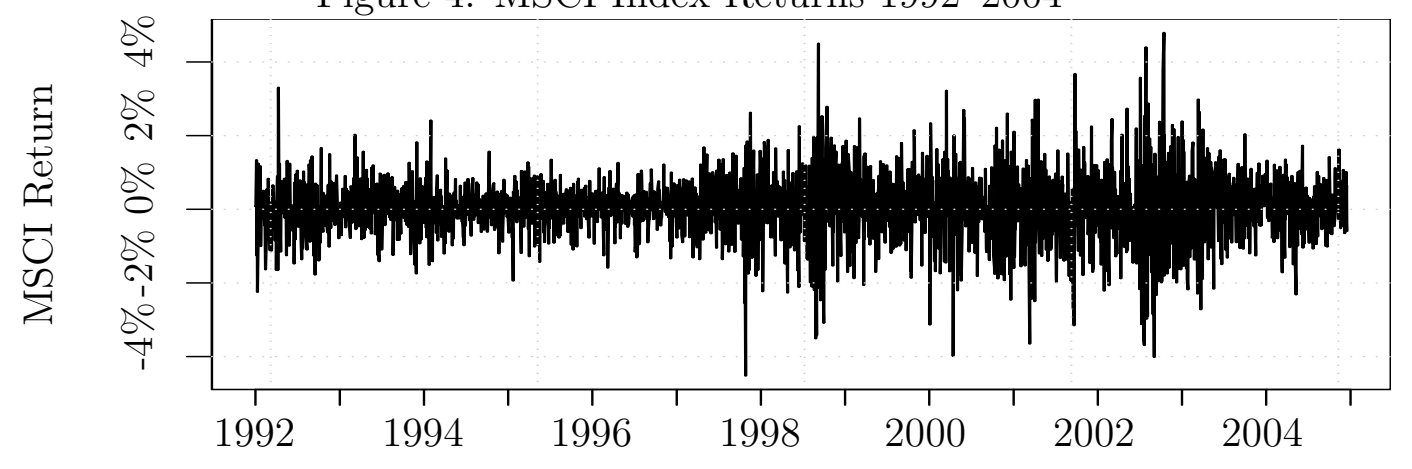


Figure 5: Interest rate spread 1992-2004

Japanese one month LIBOR minus the US one month LIBOR.

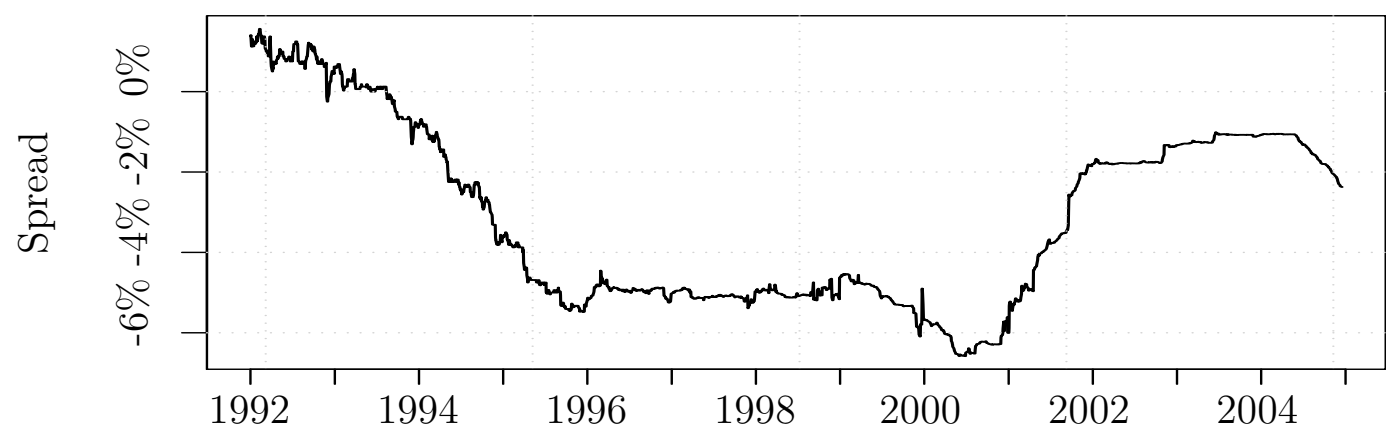

Figure 6: Simulated Cumulative prices for Model $C$, full sample

Simulated sample statistics from Model $C$ with parameter estimates from Table 2, and 1000 simulations.

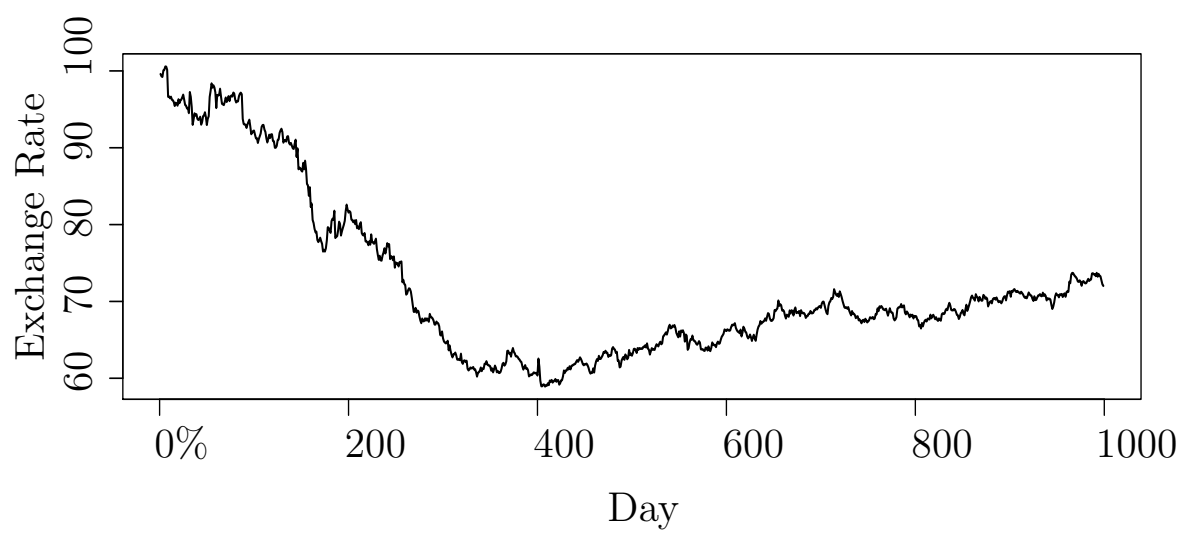


Figure 7: Simulated Cumulative prices for Model $C$, second subsample Simulated sample statistics from Model $C$ with parameter estimates from Table 2, and 1000 simulations. Parameters from the second subsample, 1997-1999.

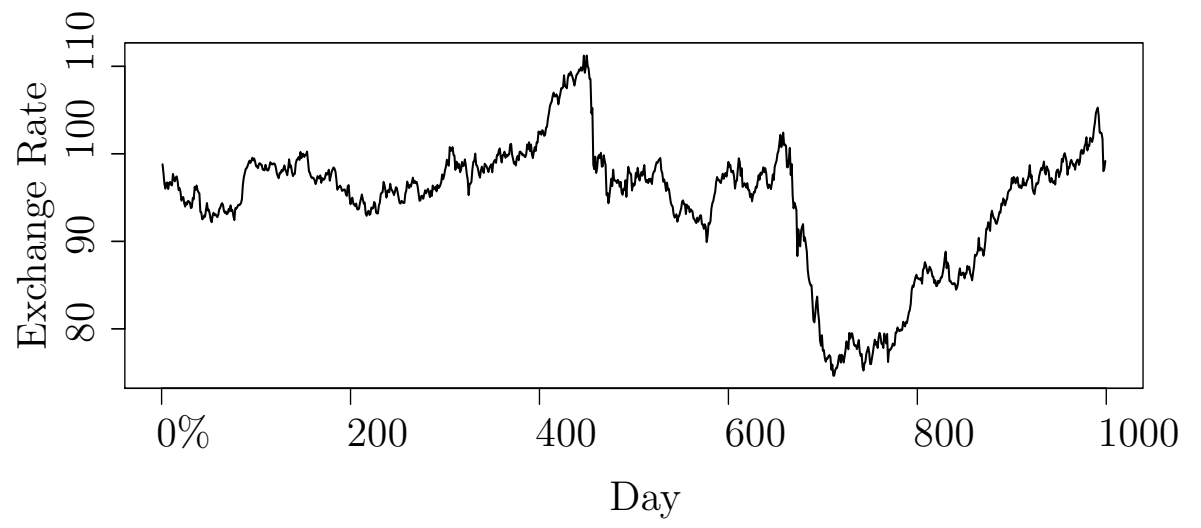




\section{References}

Abreu, D. and Brunnermeier, M. (2003). Bubbles and crashes. Econometrica, 71:173-204.

Ahn, D.-H., Dittmar, R. F., and Gallant, A. R. (2002). Quadratic term structure models: Theory and evidence. Review of Financial Studies, 15:243288.

Amihud, Y., Mendelson, H., and Pedersen, L. (2005). Liquidity and asset prices. Foundations and Trends in Finance, 1:269-364.

Angeletos, G. M., Hellwig, C., and Pavan, A. (2006). Learning, multiplicity and timing of attacks in a dynamic global game of regime change. mimeo.

Angeletos, G. M. and Werning, I. (2005). Crises and prices: Information aggregation, multiplicity, and volatility. American Economic Review, forthcoming.

Bansal, R. and Zhou, H. (2002). Term structure of interest rates with regime shifts. Journal of Finance, 57:1997-2043.

Chernov, M., Gallant, A. R., Ghysels, E., and Tauchen, G. (2003). Alternative models for stock price dynamics. Journal of Econometrics, forthcoming.

Chernov, M. and Ghysels, E. (2000). A study towards a unified approach to the joint estimation of objective and risk neutral measures for the purpose of options valuation. Journal of Financial Economics, 56(3):407-458.

Chernozhukov, V. and Hong, H. (2003). An MCMC approach to classical estimation. Journal of Econometrics, 115:293-346.

Chung, C.-S. and Tauchen, G. (2001). Testing target-zone models using efficient method of moments. Journal of Business and Economic Statistics, 19(3):255-269.

Clark, P. (1973). A subordinated stochastic process model with finite variance for speculative prices. Econometrica, 41:135-155.

Dai, Q. and Singleton, K. (2000). Specification analysis of affine term structure models. Journal of Finance, LV:1943-1978.

Daníelsson, J. and Payne, R. (2002). Liquidity determination in an order driven market. London School of Economics, www.RiskResearch.org. 
Daníelsson, J. and Shin, H. S. (2003). Endogenous risk. In Modern Risk Management - A History. Risk Books.

Dasgupta, A. (2007). Coordination and delay in global games. Journal of Economic Theory, forthcoming.

Evans, M. D. and Lyons, R. K. (2002). Order flow and exchange rate dynamics. Journal of Political Economy, 110(1):170-180.

Foster, F. D. and Viswanathan, S. (1995). Can speculative trading explain the volume-volatility relation? Journal of Business and Economic Statistics, 13:379-396.

Gallant, A. R. and Long, J. R. (1997). Estimating stochastic differential equations efficiently by minimum chi-squared. Biometrika, 84:125-141.

Gallant, A. R. and Tauchen, G. (1996). Which moments to match. Econometric Theory, 12:657-81.

Gallant, A. R. and Tauchen, G. (2002). Simulated score methods and indirect inference for continuous-time models. Chapter in preparation for the Handbook of Financial Econometrics.

Gallant, A. R. and Tauchen, G. (2006). SNP: A program for parametric time series analysis, version 9.0. User's Guide.

Goldstein, I. and Pauzner, A. (2005). Demand deposit contracts and the probability of bank runs. Journal of Finance, 60:1293-1327.

Grossman, S. J. and Miller, M. H. (1988). Liquidity and market structure. Journal of Finance, 43:617-633.

Hellwig, C., Mukherji, A., and Tsyvinski, A. (2006). Self-fulfilling currency crises: The role of interest rates. American Economic Review, forthcoming.

Jeanne, O. and Masson, P. (2000). Currency crises, sunspots and Markovswitching regimes. Journal of International Economics, 50:327-350.

Kyle, A. (1985). Continuous auctions and insider trading. Econometrica, 53 (6):1315-1335.

Metz, C. E. and Michaelis, J. (2003). The role of information disparity in the Mexican Peso crisis 1994/95: Empirical evidence. Mimeo Goethe University and University of Kassel. 
Morris, S. and Shin, H. S. (1998). Unique equilibrium in a model of selffullling currency attacks. American Economic Review, 88:587 - 597.

Morris, S. and Shin, H. S. (2000). Market risk with interdependent choice. mimeo London school of Economics.

Morris, S. and Shin, H. S. (2004). Liquidity black holes. Review of Finance, $8: 1-18$.

Obstfeld, M. (1986). Rational and self-fulfilling balance-of-payments crisis. American Economic Review, 76:i72-81.

Pastor, L. and Stambaugh, R. (2003). Liquidity risk and expected stock returns. Journal of Political Economy, 111:642-685.

Prati, A. and Sbracia, M. (2002). Currency crisis and uncertainty about fundamentals. Technical report, IMF working paper WP/02/03.

Tarashev, N. (2003). Currency crises and the informational role of interest rates. BIS Discussion Paper.

Tauchen, G. (2005). Stochastic volatility in general equilibrium.

Taylor, S. J. (1986). Modelling Financial Time Series. John Wiley.

Tillmann, P. (2002). Information disparities and the probability of currency crisis: Empirical evidence. Mimeo, University of Bonn. 